\title{
On relative $t$-designs in polynomial association schemes
}

\author{
Eiichi Bannai \\ Department of Mathematics \\ Shanghai Jiao Tong University \\ Shanghai 200240, China \\ bannai@sjtu.edu.cn \\ Sho Suda \\ Department of Mathematics Education \\ Aichi University of Education \\ Kariya 448-8542, Japan \\ suda@auecc.aichi-edu.ac.jp
}

\author{
Etsuko Bannai \\ Misakigaoka 2-8-21 \\ Itoshima 819-1136 \\ Japan \\ et-ban@rc4.so-net.ne.jp \\ Hajime Tanaka \\ Research Center for \\ Pure and Applied Mathematics \\ Graduate School of Information Sciences \\ Tohoku University \\ Sendai 980-8579, Japan \\ htanaka@m.tohoku.ac.jp
}

Submitted: Dec 8, 2014; Accepted: Dec 16, 2015; Published: Dec 23, 2015

Mathematics Subject Classifications: 05E30, 05B30

\begin{abstract}
Motivated by the similarities between the theory of spherical $t$-designs and that of $t$-designs in $Q$-polynomial association schemes, we study two versions of relative $t$-designs, the counterparts of Euclidean $t$-designs for $P$ - and/or $Q$-polynomial association schemes. We develop the theory based on the Terwilliger algebra, which is a noncommutative associative semisimple $\mathbb{C}$-algebra associated with each vertex of an association scheme. We compute explicitly the Fisher type lower bounds on the sizes of relative $t$-designs, assuming that certain irreducible modules behave nicely. The two versions of relative $t$-designs turn out to be equivalent in the case of the Hamming schemes. From this point of view, we establish a new algebraic characterization of the Hamming schemes.
\end{abstract}

Keywords: Relative $t$-design; Fisher type inequality; Terwilliger algebra

\section{Introduction}

Design theory is concerned with finding "good" finite sets that "approximate globally" their underlying spaces (often) having strong symmetry/regularity, such as the Euclidean 
space $\mathbb{R}^{n}$, the unit sphere $S^{n-1} \subseteq \mathbb{R}^{n}$, and the set of $k$-subsets of a given $v$-set. It has therefore a vast range of applications in various fields of science. See, e.g., [8, 2].

The similarities between the theories of spherical $t$-designs and combinatorial $t$ - $(v, k, \lambda)$ designs are well known; cf. [14, 13, 18, 1]. Historically, the concept of spherical $t$-designs was introduced by Delsarte, Goethals, and Seidel [14] as a continuous analogue of that of $t$-designs in $Q$-polynomial association schemes due to Delsarte [10, 11]. (Combinatorial $t-(v, k, \lambda)$ designs are precisely the $t$-designs in the Johnson scheme $J(v, k)$.) It was then generalized to the concept of Euclidean $t$-designs by Neumaier and Seidel [22], and Euclidean $t$-designs quickly became an active area of research; cf. [2]. Although the counterparts of Euclidean $t$-designs in the theory of $Q$-polynomial association schemes were already defined and discussed to some extent by Delsarte [12] (cf. [3]) much earlier as relative $t$-designs, it seems that the theory of the latter has not been fully developed yet (except in the case of the binary Hamming scheme $H(n, 2)$, in which case relative $t$-designs turn out to be equivalent to regular $t$-wise balanced designs). This paper is a contribution to this theory. Our discussions also include a concept of relative $t$-designs in general $P$-polynomial association schemes as well, following Delsarte and Seidel [15].

We refer the reader to $[10,5,6,18,21,9]$, etc., for the background on association schemes and some fundamental concepts. Throughout the paper, let $\mathfrak{X}=\left(X,\left\{R_{r}\right\}_{r=0}^{d}\right)$ be a (symmetric) $d$-class association scheme, and fix a base vertex $u_{0} \in X$. Let $X_{r}=$ $\left\{x \in X \mid\left(u_{0}, x\right) \in R_{r}\right\}$ for $r=0,1, \ldots, d$. We call $X_{0}, X_{1}, \ldots, X_{d}$ the shells of $\mathfrak{X}$. Let $\mathcal{F}(X)$ be the vector space consisting of all the real valued functions on $X$. In the following arguments we often identify $\mathcal{F}(X)$ with the vector space $\mathbb{R}^{X}$ consisting of the real column vectors with coordinates indexed by $X$.

We first introduce a concept of $t$-designs for general $P$-polynomial association schemes. Suppose that $\mathfrak{X}$ is $P$-polynomial with respect to the ordering $R_{0}, R_{1}, \ldots, R_{d}$. In the study of spherical/Euclidean $t$-designs in $\mathbb{R}^{n}$, we work with the vector space of polynomials in $n$ variables, in particular with the subspaces of homogeneous polynomials. For the $P$-polynomial scheme $\mathfrak{X}$, it is natural to consider the following subspaces of $\mathcal{F}(X)$. For every $z \in X_{j}$, we define $f_{z} \in \mathcal{F}(X)$ by

$$
f_{z}(x)= \begin{cases}1, & \text { if } x \in X_{i}, i \geqslant j, \text { and }(x, z) \in R_{i-j}, \quad(x \in X) . \\ 0, & \text { otherwise, }\end{cases}
$$

In other words, $f_{z}(x)=1$ if and only if $z$ lies on a geodesic between $u_{0}$ and $x$ in the corresponding distance-regular graph $\left(X, R_{1}\right)$. Let $\operatorname{Hom}_{j}(X)=\operatorname{span}\left\{f_{z} \mid z \in X_{j}\right\} \quad(j=$ $0,1, \ldots, d)$. Then,

$$
\operatorname{dim}\left(\operatorname{Hom}_{j}(X)\right)=\left|X_{j}\right|=: k_{j} \quad(j=0,1, \ldots, d),
$$

and we have the following direct sum decomposition of $\mathcal{F}(X)$ :

$$
\mathcal{F}(X)=\operatorname{Hom}_{0}(X)+\operatorname{Hom}_{1}(X)+\cdots+\operatorname{Hom}_{d}(X) .
$$

We now consider a (positive) weighted subset $(Y, w)$ of $X$, that is to say, a pair of a subset $Y$ of $X$ and a function $w: Y \rightarrow(0, \infty)$. Let $\left\{r_{1}, r_{2}, \ldots, r_{p}\right\}=\left\{r \mid Y \cap X_{r} \neq \emptyset\right\}$, and 
let $Y_{r_{i}}=Y \cap X_{r_{i}}, w\left(Y_{r_{i}}\right)=\sum_{y \in Y_{r_{i}}} w(y)$ for $i=1,2, \ldots, p$. We say that $Y$ is supported by the union $S=X_{r_{1}} \cup X_{r_{2}} \cup \cdots \cup X_{r_{p}}$ of $p$ shells. For any subspace $R(X)$ of $\mathcal{F}(X)$, we write $R(S)=\left\{\left.f\right|_{S} \mid f \in R(X)\right\}$.

Definition 1.1 ( $P$-polynomial case). A weighted subset $(Y, w)$ of $X$ is a relative $t$-design of $\mathfrak{X}$ with respect to $u_{0}$ if

$$
\sum_{i=1}^{p} \frac{w\left(Y_{r_{i}}\right)}{k_{r_{i}}} \sum_{x \in X_{r_{i}}} f(x)=\sum_{y \in Y} w(y) f(y)
$$

for every $f \in \operatorname{Hom}_{0}(X)+\operatorname{Hom}_{1}(X)+\cdots+\operatorname{Hom}_{t}(X)$.

This definition is due to Delsarte and Seidel [15, Section 6] for the binary Hamming scheme $H(d, 2)$. In this paper, we mostly consider the case $t=2 e$ for simplicity.

Theorem $1.2([15])$. Let $(Y, w)$ be a relative 2e-design of a Hamming scheme $H(d, q)$ with respect to $u_{0}$ in the sense of Definition 1.1. Let $S=X_{r_{1}} \cup \cdots \cup X_{r_{p}}$ be the union of the shells which support $Y$. Then,

$$
|Y| \geqslant \operatorname{dim}\left(\operatorname{Hom}_{0}(S)+\operatorname{Hom}_{1}(S)+\cdots+\operatorname{Hom}_{e}(S)\right) .
$$

Delsarte and Seidel [15] proved Theorem 1.2 only for $H(d, 2)$, but their proof works for general $q$. Theorem 1.2 also follows from Theorem 1.4 and Proposition 1.5 below. Recently, Xiang [34] succeeded in determining the right hand side of (1.1) explicitly for $H(d, 2)$, which was left open in [15]. Namely, he proved

$$
\operatorname{dim}\left(\operatorname{Hom}_{0}(S)+\operatorname{Hom}_{1}(S)+\cdots+\operatorname{Hom}_{e}(S)\right)=k_{e}+k_{e-1}+\cdots+k_{e-p+1},
$$

under a reasonable additional condition which avoids the triviality. In this paper, we focus on generalizing (1.2) to other classes of $P$-polynomial association schemes (without necessarily reference to Theorem 1.2 itself). In Appendix A, we do, however, show that Theorem 1.2 is valid for dual polar schemes as well.

The concept of relative $t$-designs for $Q$-polynomial association schemes was introduced by Delsarte [12]. We now recall the definition. Suppose that $\mathfrak{X}$ is $Q$-polynomial with respect to the ordering $E_{0}, E_{1}, \ldots, E_{d}$ of its primitive idempotents, and let $L_{j}(X)(\subseteq \mathcal{F}(X))$ be the column space of $E_{j}(j=0,1, \ldots, d)$. Then,

$$
\operatorname{dim}\left(L_{j}(X)\right)=\operatorname{rank}\left(E_{j}\right)=: m_{j} \quad(j=0,1, \ldots, d),
$$

and we have the following orthogonal direct sum decomposition of $\mathcal{F}(X)$ :

$$
\mathcal{F}(X)=L_{0}(X) \perp L_{1}(X) \perp \cdots \perp L_{d}(X) .
$$

Definition 1.3 (Q-polynomial case). A weighted subset $(Y, w)$ of $X$ is a relative $t$-design of $\mathfrak{X}$ with respect to $u_{0}$ if

$$
\sum_{i=1}^{p} \frac{w\left(Y_{r_{i}}\right)}{k_{r_{i}}} \sum_{x \in X_{r_{i}}} f(x)=\sum_{y \in Y} w(y) f(y)
$$

for every $f \in L_{0}(X) \perp L_{1}(X) \perp \cdots \perp L_{t}(X)$. 
Bannai and Bannai [3] obtained the following Fisher type inequality for general $Q$ polynomial association schemes:

Theorem $1.4([3])$. Let $(Y, w)$ be a relative 2 -design of the $Q$-polynomial scheme $\mathfrak{X}$ with respect to $u_{0}$ in the sense of Definition 1.3. Let $S=X_{r_{1}} \cup \cdots \cup X_{r_{p}}$ be the union of the shells which support $Y$. Then,

$$
|Y| \geqslant \operatorname{dim}\left(L_{0}(S)+L_{1}(S)+\cdots+L_{e}(S)\right)
$$

As in the case of (1.1), it was not easy to compute the right hand side of (1.3) explicitly. The initial attempt was made by Li, Bannai, and Bannai [20] for $H(d, 2)$, but was unsuccessful in general. Then, this attempt lead Xiang to obtain a successful result in the general case for $H(d, 2)$, as it is known that the two definitions of relative $t$-designs are essentially equivalent for $H(d, 2)$. Namely, both definitions are shown to be equivalent to the geometric definition of relative $t$-designs coming from the structure of the regular semilattice associated with $H(d, 2)$; cf. [12]. The equivalence of Definition 1.1 for $H(d, 2)$ with the definition of regular $t$-wise balanced designs was pointed out by Delsarte and Seidel [15, Theorem 6.2], whereas the equivalence of Definition 1.3 for $H(d, 2)$ with the geometric definition of relative $t$-designs was established by Delsarte [12, Theorem 9.8] (see also [4]). However, we note that

Proposition 1.5. If $\mathfrak{X}$ is a Hamming scheme $H(d, q)$, then for $t=0,1, \ldots, d$,

$$
\operatorname{Hom}_{0}(X)+\operatorname{Hom}_{1}(X)+\cdots+\operatorname{Hom}_{t}(X)=L_{0}(X)+L_{1}(X)+\cdots+L_{t}(X) .
$$

Proof. Without loss of generality, we may suppose that $X=\{0,1, \ldots, q-1\}^{d}$ and $u_{0}=$ $(0,0, \ldots, 0)$. Let $z=\left(z_{1}, z_{2}, \ldots, z_{d}\right) \in X_{j}$. Note that $z$ has exactly $j$ nonzero entries, and let $\ell_{1}, \ell_{2}, \ldots, \ell_{j}$ be the corresponding coordinates. Then, it is easy to see that $f_{z}$ is the characteristic function of the subset $\left\{\left(x_{1}, x_{2}, \ldots, x_{d}\right) \in X \mid x_{\ell_{h}}=z_{\ell_{h}}(h=1,2, \ldots, j)\right\}$, which is known to be contained in $\sum_{i=0}^{j} L_{i}(X)$; see, e.g., $[11,25] .{ }^{1}$ Since both sides of (1.4) have the same dimension, we obtain the desired result.

Thus, for $H(d, q)$, relative $t$-designs in the sense of Definition 1.1 are equivalent to relative $t$-designs in the sense of Definition 1.3. This observation seems to be new for $H(d, q)$ for general $q$. As is mentioned before, for $H(d, 2)$, the result of Xiang [34] implies that the right hand side of (1.3) is also given explicitly by

$$
\operatorname{dim}\left(L_{0}(S)+L_{1}(S)+\cdots+L_{e}(S)\right)=m_{e}+m_{e-1}+\cdots+m_{e-p+1},
$$

since $m_{j}=k_{j}(j=0,1, \ldots, d)$ in this case. In a private communication, Xiang extended his main result in [34] to general $q$. Thus, the right hand side of (1.3) is also given explicitly as (1.5) for $H(d, q)$.

\footnotetext{
${ }^{1}$ In Appendix B, we give a direct proof that $f_{z}$ belongs to $\sum_{i=0}^{j} L_{i}(X)$, which does not use the theory of regular semilattices found in $[11,25]$.
} 
In this paper, we investigate to what extent the above results can be generalized to other $P$ - and/or $Q$-polynomial association schemes. In Section 2, we derive sufficient conditions that (1.2) (resp. (1.5)) holds for a $P$-polynomial (resp. $Q$-polynomial) association scheme (Theorems 2.3 and 2.7). These conditions can be readily checked for $H(d, q)$, so that we obtain different proofs of the results of Xiang mentioned above. Concerning (1.4), we first suspected that a similar result might hold for general (formally) self-dual $P$ - and $Q$-polynomial association schemes, but it turns out that this is not the case in general. Indeed, in Section 3, we show that if $\mathfrak{X}$ is formally self-dual, $P$-polynomial (and thus $Q$-polynomial), and satisfies $\operatorname{Hom}_{0}(X)+\operatorname{Hom}_{1}(X)=L_{0}(X)+L_{1}(X)$, then $\mathfrak{X}$ must be a Hamming scheme $H(d, q)$, provided that $d \geqslant 6$ (Theorem 3.2). All of these theorems are proved using the theory of the Terwilliger algebra [29, 30, 31]. See [27] for more applications of the Terwilliger algebra to design theory.

\section{Computations of the Fisher type lower bounds}

In this section and the next, we shall use some basic facts about the Terwilliger algebra. In this context, we shall work with the complex vector space $\mathbb{C}^{X}$ instead of $\mathbb{R}^{X}$, but we note that the dimensions of the various subspaces in question do not change, as they are spanned by real vectors.

We use the following notation. For every $x \in X$, let $\hat{x} \in \mathcal{F}(X)=\mathbb{C}^{X}$ be the characteristic function of the set $\{x\}$. Let $A_{0}, A_{1}, \ldots, A_{d}$ and $E_{0}, E_{1}, \ldots, E_{d}$ be (fixed orderings of) the adjacency matrices and the primitive idempotents of $\mathfrak{X}$, respectively. Let $E_{0}^{*}, E_{1}^{*}, \ldots, E_{d}^{*}$ and $A_{0}^{*}, A_{1}^{*}, \ldots, A_{d}^{*}$ be the diagonal matrices with diagonal entries $\left(E_{i}^{*}\right)_{x x}=\left(A_{i}\right)_{u_{0} x}$ and $\left(A_{i}^{*}\right)_{x x}=|X|\left(E_{i}\right)_{u_{0} x}(x \in X, i=0,1, \ldots, d)$. They form two bases of the dual Bose-Mesner algebra with respect to $u_{0}$. When we assume that $\mathfrak{X}$ is $P$ polynomial (resp. $Q$-polynomial), we understand that $A_{0}, A_{1}, \ldots, A_{d}\left(\operatorname{resp} . E_{0}, E_{1}, \ldots, E_{d}\right)$ is the $P$-polynomial ordering (resp. $Q$-polynomial ordering) and write $A=A_{1}=\sum_{i=0}^{d} \theta_{i} E_{i}$ (resp. $A^{*}=A_{1}^{*}=\sum_{i=0}^{d} \theta_{i}^{*} E_{i}^{*}$ ). The Terwilliger algebra $T$ with respect to $u_{0}$ is the subalgebra of the full matrix algebra generated by the Bose-Mesner algebra and the dual Bose-Mesner algebra. We note that $T$ is semisimple since it is closed under conjugatetransposition.

The endpoint, dual endpoint, diameter, and the dual diameter of an irreducible $T$ module $W$ are defined by $\rho(W)=\min \left\{i \mid E_{i}^{*} W \neq 0\right\}, \rho^{*}(W)=\min \left\{i \mid E_{i} W \neq 0\right\}$, $\delta(W)=\left|\left\{i \mid E_{i}^{*} W \neq 0\right\}\right|-1$, and $\delta^{*}(W)=\left|\left\{i \mid E_{i} W \neq 0\right\}\right|-1$, respectively. ${ }^{2}$ The module $W$ is called thin (resp. dual thin) if $\operatorname{dim}\left(E_{i}^{*} W\right) \leqslant 1\left(\operatorname{resp} \cdot \operatorname{dim}\left(E_{i} W\right) \leqslant 1\right)$ $(i=0,1, \ldots, d)$. There is a unique irreducible $T$-module $W$ with $\rho(W)=0$ or $\rho^{*}(W)=0$ up to isomorphism, that is to say, the primary $T$-module $\operatorname{span}\left\{\hat{u}_{0}, A_{1} \hat{u}_{0}, \ldots, A_{d} \hat{u}_{0}\right\}$; cf. [29, Lemma 3.6]. It is thin, dual thin, and has diameter and dual diameter both equal to $d$. We call $\mathfrak{X}$ thin (resp. dual thin) with respect to $u_{0}$ if every irreducible $T$-module is thin (resp. dual thin). ${ }^{3}$ The next two lemmas will be freely used in our discussions.

\footnotetext{
${ }^{2} \operatorname{In}[29,30,31], \rho(W), \rho^{*}(W), \delta(W)$, and $\delta^{*}(W)$ are called the dual endpoint, endpoint, dual diameter, and the diameter of $W$, respectively.

${ }^{3}$ We simply call $\mathfrak{X}$ thin (resp. dual thin) if it is thin (resp. dual thin) with respect to every base vertex
} 
Lemma 2.1 ([29, Lemma 3.9]). Suppose that $\mathfrak{X}$ is P-polynomial. Let $W$ be an irreducible T-module and set $\rho=\rho(W), \delta=\delta(W)$. Then, the following hold:

(i) $A E_{i}^{*} W \subseteq E_{i-1}^{*} W+E_{i}^{*} W+E_{i+1}^{*} W(i=0,1, \ldots, d)$, where $E_{-1}^{*}=E_{d+1}^{*}=0$.

(ii) $\left\{i \mid E_{i}^{*} W \neq 0\right\}=\{\rho, \rho+1, \ldots, \rho+\delta\}$.

(iii) $E_{i}^{*} A E_{j}^{*} W \neq 0$ if $|i-j|=1(i, j=\rho, \ldots, \rho+\delta)$.

(iv) If $W$ is thin, then $E_{i} W=E_{i} E_{\rho}^{*} W(i=0,1, \ldots, d)$; in particular, $W$ is dual thin and $\delta^{*}(W)=\delta$.

Lemma 2.2 ([29, Lemma 3.12]). Suppose that $\mathfrak{X}$ is $Q$-polynomial. Let $W$ be an irreducible T-module and set $\rho^{*}=\rho^{*}(W), \delta^{*}=\delta^{*}(W)$. Then, the following hold:

(i) $A^{*} E_{i} W \subseteq E_{i-1} W+E_{i} W+E_{i+1} W(i=0,1, \ldots, d)$, where $E_{-1}=E_{d+1}=0$.

(ii) $\left\{i \mid E_{i} W \neq 0\right\}=\left\{\rho^{*}, \rho^{*}+1, \ldots, \rho^{*}+\delta^{*}\right\}$.

(iii) $E_{i} A^{*} E_{j} W \neq 0$ if $|i-j|=1\left(i, j=\rho^{*}, \ldots, \rho^{*}+\delta^{*}\right)$.

(iv) If $W$ is dual thin, then $E_{i}^{*} W=E_{i}^{*} E_{\rho^{*}} W(i=0,1, \ldots, d)$; in particular, $W$ is thin and $\delta(W)=\delta^{*}$.

We note that if $\mathfrak{X}$ is $P$-polynomial then

$$
\operatorname{Hom}_{j}(X)=\operatorname{span}\left\{\left(\sum_{i=j}^{d} E_{i}^{*} A_{i-j} E_{j}^{*}\right) \hat{z} \mid z \in X_{j}\right\}=\left(\sum_{i=j}^{d} E_{i}^{*} A_{i-j} E_{j}^{*}\right) E_{j}^{*} \mathbb{C}^{X}
$$

for $j=0,1, \ldots, d$.

Theorem 2.3. Suppose that $\mathfrak{X}$ is $P$-polynomial, and let $e, r_{1}, r_{2}, \ldots, r_{p}$ be integers with $p-1 \leqslant e \leqslant r_{1}<r_{2}<\cdots<r_{p} \leqslant d$. Suppose that every irreducible T-module $W$ with $\rho(W) \leqslant e$ is thin and satisfies $\rho(W)+\delta(W) \geqslant r_{p}$. If the $p \times p$ matrix consisting of the intersection numbers $c_{i}=p_{1, i-1}^{i}(i=1,2, \ldots, d)$ defined by

$$
\left(\begin{array}{cccc}
1 & c_{r_{1}-e+p-1} & \ldots & \left(c_{r_{1}-e+p-1} \ldots c_{r_{1}-e+1}\right) \\
\vdots & \vdots & & \vdots \\
1 & c_{r_{p}-e+p-1} & \ldots & \left(c_{r_{p}-e+p-1} \ldots c_{r_{p}-e+1}\right)
\end{array}\right)
$$

(where the $(i, j)$-entry is $\left.\prod_{h=1}^{j-1} c_{r_{i}-e+p-h}\right)$ is nonsingular, then

$$
\operatorname{dim}\left(\operatorname{Hom}_{0}(S)+\operatorname{Hom}_{1}(S)+\cdots+\operatorname{Hom}_{e}(S)\right)=k_{e}+k_{e-1}+\cdots+k_{e-p+1},
$$

where $S=X_{r_{1}} \cup X_{r_{2}} \cup \cdots \cup X_{r_{p}}$.

$u_{0} \in X$. 
Proof. Fix a set $\mathcal{W}$ of irreducible $T$-modules in $\mathbb{C}^{X}$ such that $\mathbb{C}^{X}=\bigoplus_{W \in \mathcal{W}} W$. Observe that

$$
E_{j}^{*} \mathbb{C}^{X}=\bigoplus_{\substack{W \in \mathcal{W} \\ \rho(W) \leqslant j}} E_{j}^{*} W \quad(j=0,1, \ldots, d),
$$

so that by (2.1) we have

$$
\begin{aligned}
\operatorname{Hom}_{j}(S) & =\left(\sum_{i=1}^{p} E_{r_{i}}^{*} A_{r_{i}-j} E_{j}^{*}\right) E_{j}^{*} \mathbb{C}^{X} \\
& =\bigoplus_{\substack{W \in \mathcal{W} \\
\rho(W) \leqslant j}}\left(\sum_{i=1}^{p} E_{r_{i}}^{*} A_{r_{i}-j} E_{j}^{*}\right) E_{j}^{*} W \quad(j=0,1, \ldots, e) .
\end{aligned}
$$

In particular, it follows that

$$
\sum_{j=0}^{e} \operatorname{Hom}_{j}(S)=\bigoplus_{\substack{W \in \mathcal{W} \\ \rho(W) \leqslant e}}\left(\sum_{j=0}^{e} \operatorname{Hom}_{j}(S)\right) \cap W
$$

and that

$$
\left(\sum_{j=0}^{e} \operatorname{Hom}_{j}(S)\right) \cap W=\sum_{j=\rho(W)}^{e}\left(\sum_{i=1}^{p} E_{r_{i}}^{*} A_{r_{i}-j} E_{j}^{*}\right) E_{j}^{*} W \subseteq \sum_{i=1}^{p} E_{r_{i}}^{*} W
$$

for every $W \in \mathcal{W}$ with $\rho(W) \leqslant e$.

Pick any $W \in \mathcal{W}$ with $\rho:=\rho(W) \leqslant e$, and let $v$ be a nonzero vector in $E_{\rho}^{*} W$. Recall that $\left\{i \mid E_{i}^{*} W \neq 0\right\}=\{\rho, \ldots, \rho+\delta\}$, where $\delta=\delta(W)$. First, suppose that $\rho \leqslant e-p+1$. Since $W$ is thin and since $\rho+\delta \geqslant e$, for $j=e-p+1, \ldots, e$, the vector $v_{j}=E_{j}^{*} A^{j-\rho} v$ is nonzero and hence is a basis of $E_{j}^{*} W$. Moreover, for $j=e-p+1, \ldots, e$, it follows that

$$
\begin{aligned}
\left(\sum_{i=1}^{p} E_{r_{i}}^{*} A_{r_{i}-j} E_{j}^{*}\right) v_{j} & =\sum_{i=1}^{p} \frac{1}{c_{r_{i}-j} \ldots c_{2} c_{1}} E_{r_{i}}^{*} A^{r_{i}-j} E_{j}^{*} v_{j} \\
& =\sum_{i=1}^{p} \frac{1}{c_{r_{i}-j} \ldots c_{2} c_{1}} E_{r_{i}}^{*} A^{r_{i}-\rho} v \\
& =\sum_{i=1}^{p} \frac{1}{c_{r_{i}-j} \ldots c_{2} c_{1}} E_{r_{i}}^{*} A^{r_{i}-e+p-1} E_{e-p+1}^{*} A^{e-p+1-\rho} v \\
& =\sum_{i=1}^{p}\left(c_{r_{i}-e+p-1} \ldots c_{r_{i}-j+1}\right) E_{r_{i}}^{*} A_{r_{i}-e+p-1} v_{e-p+1},
\end{aligned}
$$

where we have used the fact that $c_{n} \ldots c_{2} c_{1}$ is the number of the geodesics between two vertices at distance $n$ (in the distance-regular graph $\left(X, R_{1}\right)$ ). Since $r_{1}, r_{2}, \ldots, r_{p} \in$ 
$\{\rho, \ldots, \rho+\delta\}$, the vectors $E_{r_{i}}^{*} A_{r_{i}-e+p-1} v_{e-p+1}(i=1,2, \ldots, p)$ are nonzero and hence form a basis of $\sum_{i=1}^{p} E_{r_{i}}^{*} W$. Thus, since the coefficient matrix (2.2) is nonsingular, the vectors $\left(\sum_{i=1}^{p} E_{r_{i}}^{*} A_{r_{i}-j} E_{j}^{*}\right) v_{j}(j=e-p+1, \ldots, e)$ also form a basis of $\sum_{i=1}^{p} E_{r_{i}}^{*} W$. It follows that $\left(\sum_{j=0}^{e} \operatorname{Hom}_{j}(S)\right) \cap W=\sum_{i=1}^{p} E_{r_{i}}^{*} W$. In particular, $\operatorname{dim}\left(\left(\sum_{j=0}^{e} \operatorname{Hom}_{j}(S)\right) \cap W\right)=$ $p$. Next, suppose that $e-p+2 \leqslant \rho \leqslant e$. Likewise, using the fact that the last $(e-\rho+1)$ columns of the matrix $(2.2)$ are linearly independent, we find that the vectors $\left(\sum_{i=1}^{p} E_{r_{i}}^{*} A_{r_{i}-j} E_{j}^{*}\right) v_{j}(j=\rho, \ldots, e)$ are linearly independent, and hence that $\operatorname{dim}\left(\left(\sum_{j=0}^{e} \operatorname{Hom}_{j}(S)\right) \cap W\right)=e-\rho+1$. Thus, it follows that

$$
\begin{aligned}
\operatorname{dim}\left(\sum_{j=0}^{e} \operatorname{Hom}_{j}(S)\right) & =\sum_{\substack{W \in \mathcal{W} \\
\rho(W) \leqslant e}} \min \{p, e-\rho(W)+1\} \\
& =\sum_{\substack{W \in \mathcal{W} \\
\rho(W) \leqslant e}} \sum_{j=e-p+1}^{e} \operatorname{dim}\left(E_{j}^{*} W\right) \\
& =\sum_{j=e-p+1}^{e} \operatorname{dim}\left(E_{j}^{*} \mathbb{C}^{X}\right) \\
& =\sum_{j=e-p+1}^{e} k_{j},
\end{aligned}
$$

as desired.

Remark 2.4. In view of [7, Lemma 5.1], the assumption $\rho(W)+\delta(W) \geqslant r_{p}$ in Theorem 2.3 holds provided that $r_{p} \leqslant d-e$.

Example 2.5. Suppose that $\mathfrak{X}$ is a Hamming scheme $H(d, q)$. Then, $c_{i}=i(i=$ $1,2, \ldots, d)$. Thus, it follows that the matrix (2.2) is essentially Vandermonde (in the variables $\left.r_{1}, r_{2}, \ldots, r_{p}\right)$, and hence is nonsingular. We note that $\mathfrak{X}$ is thin; cf. [31, Example 6.1].

Example 2.6. Suppose that $\mathfrak{X}$ is a dual polar scheme. Then, $c_{i}=\left(q^{i}-1\right) /(q-1)$ $(i=1,2, \ldots, d)$ for some prime power $q \geqslant 2$. Thus, the matrix $(2.2)$ is again essentially Vandermonde (in the variables $q^{r_{1}}, q^{r_{2}}, \ldots, q^{r_{p}}$ ), and hence is nonsingular. We note that $\mathfrak{X}$ is thin; cf. [31, Example 6.1]. In Appendix A, we show that Theorem 1.2 is valid for dual polar schemes.

Next, we move on to the $Q$-polynomial case.

Theorem 2.7. Suppose that $\mathfrak{X}$ is $Q$-polynomial, and let $e, r_{1}, r_{2}, \ldots, r_{p}$ be integers with $p-1 \leqslant e \leqslant d$ and $0 \leqslant r_{1}<r_{2}<\cdots<r_{p} \leqslant d$. If every irreducible $T$-module $W$ with $\rho^{*}(W) \leqslant e$ is dual thin, and satisfies $\rho^{*}(W)+\delta^{*}(W) \geqslant e$ and $\left|\left\{i \mid E_{r_{i}}^{*} W \neq 0\right\}\right| \geqslant$ $\min \left\{p, e-\rho^{*}(W)+1\right\}$, then

$$
\operatorname{dim}\left(L_{0}(S)+L_{1}(S)+\cdots+L_{e}(S)\right)=m_{e}+m_{e-1}+\cdots+m_{e-p+1},
$$

where $S=X_{r_{1}} \cup X_{r_{2}} \cup \cdots \cup X_{r_{p}}$. 
Proof. Again, fix a set $\mathcal{W}$ of irreducible $T$-modules in $\mathbb{C}^{X}$ such that $\mathbb{C}^{X}=\bigoplus_{W \in \mathcal{W}} W$. Observe that

$$
L_{j}(X)=E_{j} \mathbb{C}^{X}=\bigoplus_{\substack{W \in \mathcal{W} \\ \rho^{*}(W) \leqslant j}} E_{j} W \quad(j=0,1, \ldots, d)
$$

so that

$$
L_{j}(S)=\left(\sum_{i=1}^{p} E_{r_{i}}^{*}\right) E_{j} \mathbb{C}^{X}=\bigoplus_{\substack{W \in \mathcal{W} \\ \rho^{*}(W) \leqslant j}}\left(\sum_{i=1}^{p} E_{r_{i}}^{*}\right) E_{j} W \quad(j=0,1, \ldots, d) .
$$

In particular, it follows that

$$
\sum_{j=0}^{e} L_{j}(S)=\bigoplus_{\substack{W \in \mathcal{W} \\ \rho^{*}(W) \leqslant e}}\left(\sum_{j=0}^{e} L_{j}(S)\right) \cap W
$$

and that

$$
\left(\sum_{j=0}^{e} L_{j}(S)\right) \cap W=\left(\sum_{i=1}^{p} E_{r_{i}}^{*}\right) \sum_{j=\rho^{*}(W)}^{e} E_{j} W \subseteq \sum_{i=1}^{p} E_{r_{i}}^{*} W
$$

for every $W \in \mathcal{W}$ with $\rho^{*}(W) \leqslant e$.

Pick any $W \in \mathcal{W}$ with $\rho^{*}:=\rho^{*}(W) \leqslant e$, and let $v$ be a nonzero vector in $E_{\rho^{*}} W$. First, suppose that $\rho^{*} \leqslant e-p+1$. Then, $v, A^{*} v, \ldots, A^{* p-1} v \in \sum_{j=\rho^{*}}^{e} E_{j} W$. Since $W$ is dual thin, $\left\{E_{i}^{*} v \mid E_{i}^{*} W \neq 0\right\}$ is an orthogonal basis of $W$. We note that $E_{r_{i}}^{*} W \neq 0$ for $i=1,2, \ldots, p$. Thus, the vectors $\left(\sum_{i=1}^{p} E_{r_{i}}^{*}\right) A^{* h} v=\sum_{i=1}^{p} \theta_{r_{i}}^{* h} E_{r_{i}}^{*} v(h=0,1, \ldots, p-$ 1) belong to $\left(\sum_{j=0}^{e} L_{j}(S)\right) \cap W$ and form a basis of $\sum_{i=1}^{p} E_{r_{i}}^{*} W$, since the coefficient matrix is Vandermonde. It follows that $\left(\sum_{j=0}^{e} L_{j}(S)\right) \cap W=\sum_{i=1}^{p} E_{r_{i}}^{*} W$. In particular, $\operatorname{dim}\left(\left(\sum_{j=0}^{e} L_{j}(S)\right) \cap W\right)=p$. Next, suppose that $e-p+2 \leqslant \rho^{*} \leqslant e$. Likewise, we find that the vectors $\left(\sum_{i=1}^{p} E_{r_{i}}^{*}\right) A^{* h} v\left(h=0,1, \ldots, e-\rho^{*}\right)$ belong to $\left(\sum_{j=0}^{e} L_{j}(S)\right) \cap W$ and are linearly independent, from which it follows that $\operatorname{dim}\left(\left(\sum_{j=0}^{e} L_{j}(S)\right) \cap W\right)=e-\rho^{*}+1$. Thus, it follows that

$$
\begin{aligned}
\operatorname{dim}\left(\sum_{j=0}^{e} L_{j}(S)\right) & =\sum_{\substack{W \in \mathcal{W} \\
\rho^{*}(W) \leqslant e}} \min \left\{p, e-\rho^{*}(W)+1\right\} \\
& =\sum_{\substack{W \in \mathcal{W} \\
\rho^{*}(W) \leqslant e}} \sum_{j=e-p+1}^{e} \operatorname{dim}\left(E_{j} W\right) \\
& =\sum_{j=e-p+1}^{e} \operatorname{dim}\left(E_{j} \mathbb{C}^{X}\right) \\
& =\sum_{j=e-p+1}^{e} m_{j},
\end{aligned}
$$


where the second equality follows since every $W \in \mathcal{W}$ with $\rho^{*}(W) \leqslant e$ is dual thin and satisfies $\left\{\rho^{*}(W), \ldots, e\right\} \subseteq\left\{\rho^{*}(W), \ldots, \rho^{*}(W)+\delta^{*}(W)\right\}=\left\{j \mid E_{j} W \neq 0\right\}$. This completes the proof.

Remark 2.8. In view of $\left[7\right.$, Lemma 7.1], the assumption $\rho^{*}(W)+\delta^{*}(W) \geqslant e$ in Theorem 2.7 holds provided that $e \leqslant\lceil d / 2\rceil$.

Example 2.9. Suppose that $\mathfrak{X}$ is a Hamming scheme $H(d, q)$. Then, $\rho(W)=\rho^{*}(W)$ for every irreducible $T$-module $W$; cf. [31, Example 6.1]. Thus, the assumption of Theorem 2.7 is satisfied provided that $e \leqslant r_{1}<r_{2}<\cdots<r_{p} \leqslant d-e$. Of course, in this case the conclusion also follows from Proposition 1.5, Theorem 2.3, and Example 2.5.

Example 2.10. Suppose that $\mathfrak{X}$ is $P$-polynomial, $Q$-polynomial, and bipartite. In this case, Caughman [7] showed that $\mathfrak{X}$ is thin, dual thin, and that every irreducible $T$-module $W$ satisfies $\delta(W)=\delta^{*}(W)=d-2 \rho^{*}(W)$ and $\rho^{*}(W) \leqslant \rho(W) \leqslant 2 \rho^{*}(W)$. Thus, the assumption of Theorem 2.7 is satisfied provided that $2 e \leqslant r_{1}<r_{2}<\cdots<r_{p} \leqslant d-e$.

Example 2.11. Suppose that $\mathfrak{X}$ is a Johnson scheme $J(v, d)$. Then, $\mathfrak{X}$ is thin, dual thin, and every irreducible $T$-module $W$ satisfies $\rho(W) \leqslant \rho^{*}(W)$; cf. [31, Example 6.1]. Thus, in view of [7, Lemma 5.1], the assumption of Theorem 2.7 is satisfied provided that $e \leqslant r_{1}<r_{2}<\cdots<r_{p} \leqslant d-e$.

We note that if some of the assumptions on the irreducible $T$-modules in Theorems 2.3 and 2.7 are not satisfied, then the dimensions of the subspaces in question can indeed be smaller. For example, we have the following result:

Proposition 2.12. Suppose that $\mathfrak{X}$ is $P$-polynomial and $Q$-polynomial, and let $S=X_{d}$. Let $\eta_{1} \geqslant \eta_{2} \geqslant \ldots \geqslant \eta_{k_{1}}$ be the eigenvalues ${ }^{4}$ of $E_{1}^{*} A E_{1}^{*}$ on $E_{1}^{*} \mathbb{C}^{X}$. For every $\theta \in \mathbb{C} \cup\{\infty\}$, let $\tilde{\theta}=-1-b_{1} /(1+\theta)$ (a Möbius transformation) where $b_{1}=p_{12}^{1}$, and define $\mu_{\theta}=\mid\{i \geqslant$ $\left.2 \mid \eta_{i}=\tilde{\theta}\right\} \mid$. Then, $\operatorname{dim}\left(\operatorname{Hom}_{0}(S)+\operatorname{Hom}_{1}(S)\right)=k_{1}-\mu_{\theta_{1}}-\mu_{\theta_{d}}$ and $\operatorname{dim}\left(L_{0}(S)+L_{1}(S)\right)=$ $m_{1}-\mu_{\theta_{d}}$.

Proof. Let $\mathcal{W}$ be as in the proofs of Theorems 2.3 and 2.7. Recall that $A$ and $A^{*}$ act on every $W \in \mathcal{W}$ as a tridiagonal pair in the sense of [19]; cf. [19, Example 1.4]. In particular, by $\left[19\right.$, Lemma 4.5] we have $\delta(W)=\delta^{*}(W)$ for $W \in \mathcal{W}$.

Let $\mathcal{W}_{1}=\left\{W \in \mathcal{W} \mid \rho(W)=1, \rho^{*}(W)=2, \delta(W)=d-2\right\}, \mathcal{W}_{d}=\{W \in \mathcal{W} \mid \rho(W)=$ $\left.1, \rho^{*}(W)=1, \delta(W)=d-2\right\}$. Let $\theta_{\mathrm{sec}}, \theta_{\min }$ be the second largest and the smallest eigenvalues of $A$, respectively. Then, in view of [17, Lemma 8.5], it follows that the condition that $\mathcal{W}_{1} \neq \emptyset\left(\right.$ resp. $\left.\mathcal{W}_{d} \neq \emptyset\right)$ implies that $\theta_{1} \in\left\{\theta_{\text {sec }}, \theta_{\min }\right\}$ (resp. $\left.\theta_{d} \in\left\{\theta_{\text {sec }}, \theta_{\min }\right\}\right)$. Next, observe that $\tilde{\theta}_{i} \notin\left(\tilde{\theta}_{\mathrm{sec}}, \tilde{\theta}_{\min }\right)(i=1,2, \ldots, d)$. On the other hand, by [17, Theorem 8.4] we have $\tilde{\theta}_{\text {sec }} \leqslant \eta_{i} \leqslant \tilde{\theta}_{\text {min }}\left(i=2,3, \ldots, k_{1}\right)$. Thus, the condition that $\mu_{\theta_{1}}>0\left(\right.$ resp. $\left.\mu_{\theta_{d}}>0\right)$ implies again that $\theta_{1} \in\left\{\theta_{\mathrm{sec}}, \theta_{\min }\right\}$ (resp. $\theta_{d} \in\left\{\theta_{\mathrm{sec}}, \theta_{\min }\right\}$ ). With these explained, it follows from [17, Lemma 8.5, Theorems 9.8, 10.1, 11.5] that every $W \in \mathcal{W}_{1} \cup \mathcal{W}_{d}$ is thin, and that $\mu_{\theta_{1}}=\left|\mathcal{W}_{1}\right|$ and $\mu_{\theta_{d}}=\left|\mathcal{W}_{d}\right|$.

\footnotetext{
${ }^{4}$ The $\eta_{i}$ are the eigenvalues of the subgraph of $\left(X, R_{1}\right)$ induced on $X_{1}$ (called the local graph), which is regular with valency $\eta_{1}=a_{1}=p_{11}^{1}$.
} 
Let $W \in \mathcal{W}$ and write $\rho=\rho(W), \rho^{*}=\rho^{*}(W)$, and $\delta=\delta(W)=\delta^{*}(W)$. Recall that $\left\{i \mid E_{i}^{*} W \neq 0\right\}=\{\rho, \ldots, \rho+\delta\}$ and $\left\{i \mid E_{i} W \neq 0\right\}=\left\{\rho^{*}, \ldots, \rho^{*}+\delta\right\}$. By [24, Theorem 1.3], we have $\operatorname{dim} E_{\rho}^{*} W=\operatorname{dim} E_{\rho^{*}} W=\operatorname{dim} E_{\rho+\delta}^{*} W=\operatorname{dim} E_{\rho^{*}+\delta} W=1$.

We first compute $\operatorname{dim}\left(\operatorname{Hom}_{0}(S)+\operatorname{Hom}_{1}(S)\right)$. Suppose that $\rho=0$. Then, $\delta=d$ and $W$ is the (thin) primary $T$-module. It follows that $\left(E_{d}^{*} A_{d} E_{0}^{*}\right) E_{0}^{*} W+\left(E_{d}^{*} A_{d-1} E_{1}^{*}\right) E_{1}^{*} W=$ $E_{d}^{*} W \neq 0$. Next, suppose that $\rho=1$. By [7, Lemma 5.1], we have $\delta \in\{d-2, d-1\}$. Observe that $\delta=d-2$ precisely when $W \in \mathcal{W}_{1} \cup \mathcal{W}_{d}$. Terwilliger [32, Lecture 34] showed that $E_{d}^{*} W=\left(E_{d}^{*} A_{d-1} E_{1}^{*}\right) E_{1}^{*} W$, from which it follows that $\left(E_{d}^{*} A_{d-1} E_{1}^{*}\right) E_{1}^{*} W=0$ if and only if $\delta=d-2$, i.e., $W \in \mathcal{W}_{1} \cup \mathcal{W}_{d}$. Thus, as in the proof of Theorem 2.3, it follows that $\operatorname{dim}\left(\operatorname{Hom}_{0}(S)+\operatorname{Hom}_{1}(S)\right)=k_{1}-\left|\mathcal{W}_{1} \cup \mathcal{W}_{d}\right|=k_{1}-\mu_{\theta_{1}}-\mu_{\theta_{d}}$.

We now compute $\operatorname{dim}\left(L_{0}(S)+L_{1}(S)\right)$. We note that $E_{d}^{*} W=E_{d}^{*} E_{\rho^{*}} W$ in view of [23, Lemma 5.1]. Suppose that $\rho^{*}=0$. Then, $\delta=d$ and $W$ is again the primary $T$-module. It follows that $E_{d}^{*}\left(E_{0} W+E_{1} W\right)=E_{d}^{*} W \neq 0$. Next, suppose that $\rho^{*}=1$. By [7, Lemma 7.1], we have $\delta \in\{d-2, d-1\}$. It follows that $E_{d}^{*} E_{1} W=0$ if and only if $W \in \mathcal{W}_{d}$. Thus, as in the proof of Theorem 2.7, it follows that $\operatorname{dim}\left(L_{0}(S)+L_{1}(S)\right)=m_{1}-\left|\mathcal{W}_{d}\right|=m_{1}-\mu_{\theta_{d}}$.

Example 2.13. Suppose that $\mathfrak{X}$ is a Hamming scheme $H(d, q)$. Then, $k_{1}=m_{1}=d(q-1)$, $b_{1}=(d-1)(q-1), \theta_{i}=q(d-i)-d(i=0,1, \ldots, d)$, and it is easy to see that $\mu_{\theta_{1}}=0$ and $\mu_{\theta_{d}}=d-1$. We note that relative 2-designs supported by $X_{d}$ (in the sense of both Definition 1.1 and Definition 1.3) are precisely the 2-designs (i.e., orthogonal arrays with strength 2) in the Hamming scheme $H(d, q-1)$ induced on $X_{d}$, and Proposition 2.12 gives the Rao bound $1+d(q-2)$.

Example 2.14. Suppose that $\mathfrak{X}$ is a Johnson scheme $J(v, d)$. Then, $k_{1}=d(v-d)$, $m_{1}=v-1, b_{1}=(d-1)(v-d-1), \theta_{i}=(d-i)(v-d-i)-i(i=0,1, \ldots, d)$, and it is easy to see that $\mu_{\theta_{1}}=(d-1)(v-d-1)$ and $\mu_{\theta_{d}}=d-1$. We note that relative 2 -designs supported by $X_{d}$ (in the sense of both Definition 1.1 and Definition 1.3) are precisely the 2-designs in the Johnson scheme $J(v-d, d)$ induced on $X_{d}$, and Proposition 2.12 gives the Fisher bound $v-d$.

\section{A characterization of Hamming schemes}

In this section, for $d \geqslant 6$, we characterize the Hamming schemes $H(d, q)$ as the formally self-dual $P$ - and $Q$-polynomial association schemes with the property that $\operatorname{Hom}_{0}(X)+$ $\operatorname{Hom}_{1}(X)=L_{0}(X)+L_{1}(X)$. We begin with the following result:

Proposition 3.1. Suppose that $\mathfrak{X}$ is P-polynomial, Q-polynomial, and that $\operatorname{Hom}_{0}(X)+$ $\operatorname{Hom}_{1}(X)=L_{0}(X)+L_{1}(X)$. Then, $c_{i} /\left(\theta_{i}^{*}-\theta_{0}^{*}\right)$ is independent of $i=1,2, \ldots, d$.

Proof. By (2.1) and since $A \hat{u}_{0} \in E_{1}^{*} \mathbb{C}^{X}$, the vector

$$
\left(\sum_{i=1}^{d} E_{i}^{*} A_{i-1} E_{1}^{*}\right) A \hat{u}_{0}=\sum_{i=1}^{d} E_{i}^{*} A_{i-1} A \hat{u}_{0}=\sum_{i=1}^{d} c_{i} A_{i} \hat{u}_{0}
$$


belongs to $\operatorname{Hom}_{1}(X) \subset L_{0}(X)+L_{1}(X)$. On the other hand, this vector is in the primary $T$-module $\operatorname{span}\left\{\hat{u}_{0}, A_{1} \hat{u}_{0}, \ldots, A_{d} \hat{u}_{0}\right\}=\operatorname{span}\left\{E_{0} \hat{u}_{0}, E_{1} \hat{u}_{0}, \ldots, E_{d} \hat{u}_{0}\right\}$. Thus, it is written as

$$
\sum_{i=1}^{d} c_{i} A_{i} \hat{u}_{0}=\alpha E_{0} \hat{u}_{0}+\beta E_{1} \hat{u}_{0}=\frac{1}{|X|} \sum_{i=0}^{d}\left(\alpha+\beta \theta_{i}^{*}\right) A_{i} \hat{u}_{0},
$$

for some $\alpha, \beta \in \mathbb{C}$. Comparing the coefficients of $\hat{u}_{0}$, we find $\beta=-\alpha / \theta_{0}^{*}$, and hence

$$
c_{i}=\frac{\alpha}{|X| \theta_{0}^{*}}\left(\theta_{0}^{*}-\theta_{i}^{*}\right) \quad(i=1,2, \ldots, d),
$$

as desired. ${ }^{5}$

Using this result, we now prove the following theorem:

Theorem 3.2. Suppose that $\mathfrak{X}$ is formally self-dual, P-polynomial (and $Q$-polynomial), and satisfies $\operatorname{Hom}_{0}(X)+\operatorname{Hom}_{1}(X)=L_{0}(X)+L_{1}(X)$. If $d \geqslant 6$, then $\mathfrak{X}$ is the Hamming scheme $H(d, q)$ for some $q$.

Proof. Since $\mathfrak{X}$ is formally self-dual, in the notation of [5, Section 3.5] and [29, Section $2]$, the parameters of $\mathfrak{X}$ satisfy one of the following cases $^{6}$ : (I) with $s=s^{*} \neq 0$; (I) with $s=s^{*}=0$; (II) with $s=s^{*}$; (IIC); and (III) with $s=s^{*}$.

First, consider Case (I) with $s=s^{*} \neq 0$. Then, it follows that

$$
\frac{c_{i}}{\theta_{i}^{*}-\theta_{0}^{*}}=\frac{q^{i}\left(1-s q^{i+d+1}\right)\left(r_{1}-s q^{i}\right)\left(r_{2}-s q^{i}\right)}{s q^{d}\left(1-s q^{i+1}\right)\left(1-s q^{2 i}\right)\left(1-s q^{2 i+1}\right)} \quad(i=1,2, \ldots, d-1),
$$

and this is independent of $i$ by Proposition 3.1, so that

$$
s q^{d}\left(1-s q^{i+1}\right)\left(1-s q^{2 i}\right)\left(1-s q^{2 i+1}\right)=\left(\theta_{1}^{*}-\theta_{0}^{*}\right) q^{i}\left(1-s q^{i+d+1}\right)\left(r_{1}-s q^{i}\right)\left(r_{2}-s q^{i}\right)
$$

for $i=1,2, \ldots, d-1$, and this identity is valid for $i=d$ as well. However, as polynomials in $q^{i}$, the left hand side is of degree five, whereas the right hand side is of degree four. Since $d \geqslant 6$, this is impossible. Case (I) with $s=s^{*}=0$ is ruled out in the same way.

Next, consider Case (II) with $s=s^{*}$. Then, it follows that

$$
\frac{c_{i}}{\theta_{i}^{*}-\theta_{0}^{*}}=\frac{(i+s+d+1)\left(i+s-r_{1}\right)\left(i+s-r_{2}\right)}{(i+1+s)(2 i+1+s)(2 i+s)} \quad(i=1,2, \ldots, d-1) .
$$

Again, as polynomials in $i$, the denominator must be a scalar multiple of the numerator. In particular, they have the same roots. Since $1+s \neq s+d+1$, we may assume that $1+s=s-r_{1}$, i.e., $r_{1}=-1$. Then, since $r_{1}+r_{2}=s+s^{*}+d+1$, we have $r_{2}=2 s+d+2$. Using this and $\left\{s+d+1, s-r_{2}\right\}=\{(1+s) / 2, s / 2\}$, it follows that $d= \pm 1 / 4$, which is absurd.

\footnotetext{
${ }^{5}$ In fact, we have $\alpha=\sum_{i=1}^{d} c_{i} k_{i}$.

${ }^{6}$ In the terminology of [33], these are of $q$-Racah, affine $q$-Krawtchouk, Racah, Krawtchouk and Bannai/Ito types, respectively.
} 
If $\mathfrak{X}$ satisfies Case (III) with $s=s^{*}$, then by the classification due to Terwilliger [28], it follows that $\mathfrak{X}$ is isomorphic to $H(d, 2)$ ( $d$ even) or the bipartite half of $H(2 d+1,2)$, but with respect to the second $P$-polynomial orderings. ${ }^{7}$ We have $c_{i}=i(i=1,2, \ldots, d)$ in either case, and it follows that $c_{i} /\left(\theta_{i}^{*}-\theta_{0}^{*}\right)$ cannot be constant, since $\theta_{0}^{*}, \theta_{1}^{*}, \ldots, \theta_{d}^{*}$ are not an arithmetic progression.

Thus, we are left with Case (IIC). In this case, by the classification due to Egawa [16], $\mathfrak{X}$ is a Hamming scheme or a Doob scheme. If $\mathfrak{X}$ is a Hamming scheme, then we are done. Thus, suppose that $\mathfrak{X}$ is a Doob scheme. Then, there is a thin irreducible $T$-module $W$ with $\rho(W)=1, \rho^{*}(W)=2$, and $\delta(W)=d-2$. This fact follows from Tanabe's description [26] of the irreducible $T$-modules of Doob schemes, but we may also prove it as follows. The local graph of the Doob graph $\left(X, R_{1}\right)$ (whose adjacency matrix is essentially $E_{1}^{*} A E_{1}^{*}$ ) is a disjoint union of hexagons and 3 -cliques, so that it has -2 as an eigenvalue. On the other hand, we have $-1-b_{1} /\left(1+\theta_{1}\right)=-2$, where $b_{1}=p_{12}^{1}$. Thus, by [17, Theorem 9.8], any eigenvector (in $E_{1}^{*} \mathbb{C}^{X}$ ) of $E_{1}^{*} A E_{1}^{*}$ with eigenvalue -2 generates such a $T$-module. Now, let $v$ be a nonzero vector in $E_{1}^{*} W$. Then, $\left(\sum_{i=1}^{d} E_{i}^{*} A_{i-1} E_{1}^{*}\right) v$ is nonzero and belongs to $\operatorname{Hom}_{1}(X)$. However, since $\rho^{*}(W)=2$, it is contained in $L_{2}(X)+$ $L_{3}(X)+\cdots+L_{d}(X)$. Thus, we conclude that $\operatorname{Hom}_{0}(X)+\operatorname{Hom}_{1}(X) \neq L_{0}(X)+L_{1}(X)$, and the proof is complete.

\section{A Comments on Theorem 1.2}

In this appendix, we generalize Theorem 1.2 to dual polar schemes (Theorem A.6). Suppose that $\mathfrak{X}$ is a dual polar scheme, so that $X$ is the set of maximal isotropic subspaces of a vector space $V$ over a finite field, equipped with a non-degenerate form (alternating, Hermitian, or quadratic) of Witt index $d$. For convenience, we shall work with the dual polar graph $\left(X, R_{1}\right)$ with path-length distance $\partial$.

Lemma A.1. Let $x, y, z \in X$. Then, $\partial(x, z)+\partial(z, y)=\partial(x, y)$ if and only if $x \cap y \subseteq$ $z=(x \cap z)+(y \cap z)$.

Proof. Immediate from $\operatorname{dim}(x \cap z)+\operatorname{dim}(y \cap z) \leqslant d+\operatorname{dim}(x \cap y \cap z) \leqslant d+\operatorname{dim}(x \cap y)$.

For the moment, fix $x, y \in X$ and write $i=\partial\left(u_{0}, x\right), j=\partial\left(u_{0}, y\right), h=\partial(x, y)$, and $\ell=\operatorname{dim}\left(u_{0} \cap U\right)$, where $U=x \cap y$. We note that $\ell \geqslant d-i-j$. Our goal is to show that $f_{x} f_{y} \in \operatorname{Hom}_{d-\ell}(X)$. We set $X^{\prime}=\{z \in X \mid U \subseteq z\}$, and observe that $X^{\prime}$ induces a dual polar graph with diameter $h$.

Lemma A.2. For every $z \in X$, there is a unique $z^{\prime} \in X^{\prime}$ such that $\partial\left(z, z^{\prime}\right)=\partial\left(z, X^{\prime}\right)$. Moreover, it holds that $\partial\left(z, z_{1}\right)=\partial\left(z, z^{\prime}\right)+\partial\left(z^{\prime}, z_{1}\right)$ for all $z_{1} \in X^{\prime}$.

Proof. Set $z^{\prime}=U+\left(z \cap U^{\perp}\right) \in X^{\prime}$. Pick any $z_{1} \in X^{\prime}$. Then, $z^{\prime}=\left(z \cap z^{\prime}\right)+\left(z_{1} \cap z^{\prime}\right)$ since $U \subseteq z_{1}$ and $z \cap U^{\perp} \subseteq z$. Moreover, $z \cap z_{1} \subseteq z \cap U^{\perp} \subseteq z^{\prime}$. Thus, $\partial\left(z, z_{1}\right)=\partial\left(z, z^{\prime}\right)+\partial\left(z^{\prime}, z_{1}\right)$ by Lemma A.1, and the result follows.

\footnotetext{
${ }^{7}$ The second $P$-polynomial ordering of the Johnson scheme $J(2 d+1, d)$ (corresponding to the Odd graph $O_{d+1}$ ) satisfies Case (III), but with $s=2 d+3$ and $s^{*}=2 d+2$.
} 
Lemma A.3. Suppose that $z \in X^{\prime}$ satisfies $f_{x}(z)=f_{y}(z)=1$. Then, $\partial\left(u_{0}, z\right)=d-\ell$.

Proof. First, $u_{0} \cap z \subseteq x$ by Lemma A.1 and since $\partial\left(u_{0}, x\right)+\partial(x, z)=\partial\left(u_{0}, z\right)$. Likewise, $u_{0} \cap z \subseteq y$. Thus, $u_{0} \cap z \subseteq u_{0} \cap U$. On the other hand, $u_{0} \cap U \subseteq u_{0} \cap z$ since $z \in X^{\prime}$. It follows that $u_{0} \cap z=u_{0} \cap U$, as desired.

Lemma A.4. For every $z \in X$ such that $f_{x}(z)=f_{y}(z)=1$, there is a unique $z^{\prime} \in X^{\prime}$ such that $f_{x}\left(z^{\prime}\right)=f_{y}\left(z^{\prime}\right)=1$ and $f_{z^{\prime}}(z)=1$.

Proof. Let $z^{\prime}\left(=U+\left(z \cap U^{\perp}\right)\right)$ be as in Lemma A.2. Then, $f_{x}\left(z^{\prime}\right)=f_{y}\left(z^{\prime}\right)=1$ and $f_{z^{\prime}}(z)=1$. To show the uniqueness, suppose that $z_{1} \in X^{\prime}$ satisfies $f_{x}\left(z_{1}\right)=f_{y}\left(z_{1}\right)=1$ and $f_{z_{1}}(z)=1$. Then, it follows from Lemma A.3 that $\partial\left(u_{0}, z^{\prime}\right)=\partial\left(u_{0}, z_{1}\right)=d-\ell$, so that $\partial\left(z, z^{\prime}\right)=\partial\left(z, z_{1}\right)$. But then, we must have $z^{\prime}=z_{1}$ by Lemma A.2, and the proof is complete.

Proposition A.5. With the above notation, it holds that

$$
f_{x} f_{y}=\sum_{\substack{z \in X^{\prime} \\ f_{x}(z)=f_{y}(z)=1}} f_{z} \in \operatorname{Hom}_{d-\ell}(X) .
$$

Proof. Immediate from Lemmas A.3 and A.4.

Theorem A.6. Theorem 1.2 is valid for dual polar schemes.

Proof. Suppose that $f \in \operatorname{Hom}_{0}(X)+\operatorname{Hom}_{1}(X)+\cdots+\operatorname{Hom}_{e}(X)$ satisfies $\left.f\right|_{Y} \equiv 0$. Then, $f^{2} \in \operatorname{Hom}_{0}(X)+\operatorname{Hom}_{1}(X)+\cdots+\operatorname{Hom}_{2 e}(X)$ by Proposition A.5. Thus,

$$
\sum_{i=1}^{p} \frac{w\left(Y_{r_{i}}\right)}{k_{r_{i}}} \sum_{x \in X_{r_{i}}}(f(x))^{2}=\sum_{y \in Y} w(y)(f(y))^{2}=0
$$

from which it follows that the restriction map $\operatorname{Hom}_{0}(S)+\operatorname{Hom}_{1}(S)+\cdots+\operatorname{Hom}_{e}(S) \rightarrow$ $\mathcal{F}(Y)\left(\left.\left.f\right|_{S} \mapsto f\right|_{Y}\right)$ is injective, and the result follows by comparing the dimensions.

\section{B Comments on Proposition 1.5}

We use the notation in the proof of Proposition 1.5. We mentioned there that the function $f_{z}$ belongs to $\sum_{i=0}^{j} L_{i}(X)$. While this fact is just a special case of a more general result about regular semilattices $[11,12,25]$, we now provide an independent proof.

We identify $\{0,1, \ldots, q-1\}$ with the additive group $\mathbb{Z} / q \mathbb{Z}$. Let $\zeta \in \mathbb{C}$ be a primitive $q^{\text {th }}$ root of unity. Then, the additive group $X$ and its dual group $X^{*}$ are isomorphic, and an isomorphism is given by $x=\left(x_{1}, x_{2}, \ldots, x_{d}\right) \mapsto \varepsilon_{x}$, where $\varepsilon_{x}(y)=\zeta^{\sum_{\ell=1}^{d} x_{\ell} y_{\ell}}$ for every $y=\left(y_{1}, y_{2}, \ldots, y_{d}\right) \in X$. In fact, it is well known (and is easily checked) that $L_{i}(X)=\operatorname{span}\left\{\varepsilon_{x} \mid x \in X_{i}\right\}$ (over $\mathbb{C}$ ) for $i=0,1, \ldots, d$, i.e., $H(d, q)$ is self-dual. 
Assume that $i>j$, and pick any $y=\left(y_{1}, y_{2}, \ldots, y_{d}\right) \in X_{i}$. Then, the (standard) Hermitian inner product between $\varepsilon_{y}$ and $f_{z}$ is given by

$$
\left(\prod_{h=1}^{j} \zeta^{z_{\ell}} y_{\ell_{h}}\right)\left(\prod_{\ell \neq \ell_{1}, \ldots, \ell_{j}}\left(\sum_{x_{\ell}=0}^{q-1} \zeta^{x_{\ell} y_{\ell}}\right)\right) .
$$

Since $i>j$, there is an $\ell \neq \ell_{1}, \ldots, \ell_{j}$ such that $y_{\ell} \neq 0$. For this $\ell$, we have $\sum_{x_{\ell}=0}^{q-1} \zeta^{x_{\ell} y_{\ell}}=0$. Thus, $f_{z}$ is orthogonal to $\varepsilon_{y}$. It follows that $f_{z}$ is orthogonal to $\sum_{i=j+1}^{d} L_{i}(X)$, and hence it is contained in $\sum_{i=0}^{j} L_{i}(X)$, as desired.

\section{Acknowledgements}

Eiichi Bannai is supported in part by NSFC grant No. 11271257. Sho Suda was supported by JSPS Research Fellowships for Young Scientists. Hajime Tanaka was supported in part by JSPS KAKENHI Grant No. 23740002 and No. 25400034.

\section{References}

[1] Ei. Bannai, Et. Bannai, Algebraic Combinatorics on Spheres, Springer-Verlag, Tokyo, 1999 (in Japanese).

[2] Ei. Bannai, Et. Bannai, A survey on spherical designs and algebraic combinatorics on spheres, European J. Combin. 30 (2009) 1392-1425.

[3] Ei. Bannai, Et. Bannai, Remarks on the concepts of $t$-designs, J. Appl. Math. Comput. 40 (2012) 195-207.

[4] Ei. Bannai, Et. Bannai, T. Ito, Introduction to Algebraic Combinatorics, in preparation (in Japanese).

[5] Ei. Bannai, T. Ito, Algebraic Combinatorics I: Association Schemes, Benjamin/Cummings, Menlo Park, CA, 1984.

[6] A.E. Brouwer, A.M. Cohen, A. Neumaier, Distance-Regular Graphs, Springer-Verlag, Berlin, 1989.

[7] J.S. Caughman, IV, The Terwilliger algebras of bipartite $P$ - and $Q$-polynomial schemes, Discrete Math. 196 (1999) 65-95.

[8] C.J. Colbourn, J.H. Dinitz (Eds.), The CRC Handbook of Combinatorial Designs (2nd edn.), CRC Press, Boca Raton, FL, 2007.

[9] E.R. van Dam, J.H. Koolen, H. Tanaka, Distance-regular graphs, preprint (2014); arXiv: 1410.6294

[10] P. Delsarte, An algebraic approach to the association schemes of coding theory, Thesis, Universite Catholique de Louvain, Philips Res. Rep. Suppl. No. 10 (1973).

[11] P. Delsarte, Association schemes and $t$-designs in regular semilattices, J. Combin. Theory Ser. A 20 (1976) 230-243. 
[12] P. Delsarte, Pairs of vectors in the space of an association scheme, Philips Res. Rep. 32 (1977) 373-411.

[13] P. Delsarte, Hahn polynomials, discrete harmonics, and t-designs, SIAM J. Appl. Math. 34 (1978) 157-166.

[14] P. Delsarte, J.M. Goethals, J.J. Seidel, Spherical codes and designs, Geom. Dedicata 6 (1977) 363-388.

[15] P. Delsarte, J.J. Seidel, Fisher type inequalities for Euclidean $t$-designs, Linear Algebra Appl. 114/115 (1989) 213-230.

[16] Y. Egawa, Characterization of $H(n, q)$ by the parameters, J. Combin. Theory Ser. A 31 (1981) 108-125.

[17] J.T. Go, P. Terwilliger, Tight distance-regular graphs and the subconstituent algebra, European J. Combin. 23 (2002) 793-816.

[18] C.D. Godsil, Algebraic Combinatorics, Chapman \& Hall, New York, 1993.

[19] T. Ito, K. Tanabe, P. Terwilliger, Some algebra related to $P$ - and $Q$-polynomial association schemes, Codes and Association Schemes (A. Barg, S. Litsyn, eds.), American Mathematical Society, Providence, RI, 2001, pp. 167-192; arXiv:math/0406556.

[20] Z. Li, Ei. Bannai, Et. Bannai, Tight relative 2- and 4-designs on binary Hamming association schemes, Graphs Combin. 30 (2014) 203-227.

[21] W.J. Martin, H. Tanaka, Commutative association schemes, European J. Combin. 30 (2009) 1497-1525; arXiv:0811.2475.

[22] A. Neumaier, J.J. Seidel, Discrete measures for spherical designs, eutactic stars and lattices, Nederl. Akad. Wetensch. Indag. Math. 50 (1988) 321-334.

[23] K. Nomura, P. Terwilliger, Sharp tridiagonal pairs, Linear Algebra Appl. 429 (2008) 79-99; arXiv:0712.3665.

[24] K. Nomura, P. Terwilliger, The structure of a tridiagonal pair, Linear Algebra Appl. 429 (2008) 1647-1662; arXiv:0802.1096.

[25] D. Stanton, Harmonics on posets, J. Combin. Theory Ser. A 40 (1985) 136-149.

[26] K. Tanabe, The irreducible modules of the Terwilliger algebras of Doob schemes, J. Algebraic Combin. 6 (1997) 173-195.

[27] H. Tanaka, New proofs of the Assmus-Mattson theorem based on the Terwilliger algebra, European J. Combin. 30 (2009) 736-746; arXiv:math/0612740.

[28] P. Terwilliger, $P$ and $Q$ polynomial schemes with $q=-1$, J. Combin. Theory Ser. B 42 (1987) 64-67.

[29] P. Terwilliger, The subconstituent algebra of an association scheme I, J. Algebraic Combin. 1 (1992) 363-388.

[30] P. Terwilliger, The subconstituent algebra of an association scheme II, J. Algebraic Combin. 2 (1993) 73-103.

[31] P. Terwilliger, The subconstituent algebra of an association scheme III, J. Algebraic Combin. 2 (1993) 177-210. 
[32] P. Terwilliger, The subconstituent algebra of a graph, the thin condition, and the $Q$-polynomial property, unpublished lecture notes (1993).

[33] P. Terwilliger, Two linear transformations each tridiagonal with respect to an eigenbasis of the other; comments on the parameter array, Des. Codes Cryptogr. 34 (2005) 307-332; arXiv:math/0306291.

[34] Z. Xiang, A Fisher type inequality for weighted regular $t$-wise balanced designs, J. Combin. Theory Ser. A 119 (2012) 1523-1527. 